\title{
Chaos for successive maxima map implies chaos for the original map
}

\author{
Abraham Boyarsky - Peyman Eslami - Paweł Góra · Zhenyang Li · \\ Jonathan Meddaugh • Brian E. Raines
}

Received: date / Accepted: date

\begin{abstract}
$\tau$ is a continuous map on a metric compact space $X$. For a continuous function $\phi: X \rightarrow \mathbb{R}$ we consider a 1-dimensional map $T$ (possibly multi-valued) which sends a local $\phi$-maximum on $\tau$ trajectory to the next one: consecutive maxima map. The idea originated with famous Lorenz's paper on strange attractor. We prove that if $T$ has a horseshoe disjoint from fixed points, then $\tau$ is in some sense chaotic, i.e., it has a turbulent trajectory and thus a continuous invariant measure.
\end{abstract}

\footnotetext{
Abraham Boyarsky

Department of Mathematics and Statistics, Concordia University, 1455 de Maisonneuve Blvd. West, Montreal, Quebec H3G 1M8, Canada

E-mail: abraham.boyarsky@concordia.ca

Peyman Eslami

Dipartimento di Matematica, Universita' di Roma Tor Vergata, Via della Ricerca Scientifica, I-00133 Roma, Italy

E-mail: eslami@axp.mat.uniroma2.it

Paweł Góra

Department of Mathematics and Statistics, Concordia University, 1455 de Maisonneuve Blvd. West, Montreal, Quebec H3G 1M8, Canada

Tel.: +514-848-2424, ext. 3257

Fax: +514-848-2831

E-mail: pawel.gora@concordia.ca

Zhenyang Li

Department of Mathematics and Statistics, Concordia University, 1455 de Maisonneuve Blvd. West, Montreal, Quebec H3G 1M8, Canada

E-mail: zhenyangemail@gmail.com

Jonathan Meddaugh Department of Mathematics, Baylor University, Waco, TX 76798-7328,USA

E-mail: jonathan_meddaugh@baylor.edu · Brian E. Raines Department of Mathematics, Baylor University, Waco, TX 76798-7328,USA

E-mail: brian_raines@baylor.edu
}

Keywords Successive maxima map - Lorenz system · Turbulent trajectories · Chaotic behaviour

Mathematics Subject Classification (2000) MSC $37 \mathrm{M} 10 \cdot \mathrm{MSC} 37 \mathrm{~B} 20 \cdot \mathrm{MSC} 37 \mathrm{~N} 10$

\section{Introduction}

The main goal of the paper is to study when and how a lower dimensional projection of a higher dimensional chaotic system inherits the turbulent dynamics. The literature on chaotic dynamical systems is very rich. We give references $[1,3,5,9,12,20,22]$, for example. The problem of inheriting chaoticity seems not to be studied in depth before. Our methods allow to consider the case when the lower dimensional projection is actually a setvalued map and not a well defined transformation.

The idea of using successive maxima of one variable in a higher dimensional dynamical system was introduced by Lorenz in his numerical studies of a 3dimensional system of differential equations that model atmospheric effects [16]. Plotting the successive maxima of $z$-component he discovered a simple unimodal functional relationship $T$ between one maxima and the previous one. Since the orbits of such 3 dimensional system maps looked chaotic, the unproved conclusion was that the one-dimensional map for successive maxima was a simple indicator of that chaos. Indeed, the successive maxima map was modeled by a piecewise expanding map which is exact [12, p.125]. This gave some credence to the hypothesis that the overall system must be chaotic. However, the connection between ergodic properties of the successive maxima map and the original map have never been studied theoretically. It is the purpose of this note to investigate such a connection. 
In section 2 we start with a discrete time one dimensional map $\tau$ and define the map of successive maxima $\bar{\tau}_{M}$. We prove that there there is an interesting relationship between the maxima map and the first return map $\tau_{A}$, namely that $\bar{\tau}_{M} \circ \tau=\tau \circ \tau_{A}$. From this it follows that if $\bar{\tau}_{M}$ behaves chaotically then so must $\tau$. In section 3 we prove our main result: if the successive maxima multi-valued map is chaotic in an appropriate sense then so is the original higher dimensional map. We show that if the observed 1-dimensional map $T$ (possibly multi-valued) has a horseshoe or generalized horseshoe (Definitions 5 and 6) whose support is disjoint with the images of the fixed points of the original transformation $\tau$, then $\tau$ has a turbulent trajectory. In section 4 we show that the main result of section 3 cannot be improved. In section 5 we prove the existence of a continuous invariant measure for the original map $\tau$. In section 6 we present a number of examples.

\section{Successive maxima map}

In this section we introduce the successive maxima map, the main object and tool of our investigation.

Let $X$ be a compact metric space and $\tau: X \rightarrow$ $X$ a continuous transformation. Let $\phi: X \rightarrow \mathbb{R}$ be a continuous function.

Define $A$ as follows:

$$
A=\left\{x \in X \mid \phi(x) \leq \phi(\tau(x)) \geq \phi\left(\tau^{2}(x)\right)\right\} .
$$

Let $M=\tau(A)$. We call $M$ the set of relative $\phi$-maxima of trajectories of $\tau$. Both $A$ and $M$ are compact subsets of $X$. Both $A$ and $M$ depend on $\tau$ and $\phi$, but we suppress this dependence in the notation whenever it doesn't cause confusion.

Definition 1 (Background successive maxima map) The map $\bar{\tau}_{M}: M \rightarrow M$ defined by $\bar{\tau}_{M}(y)=\tau^{k}(y)$, where $k=k(y)=\min \left\{j \geq 2 \mid \tau^{j-1}(y) \in A\right\}$ is called the background successive $\phi$-maxima map of $\tau$.

Remark 1 If $n(y)=\min \left\{j \geq 1 \mid \tau^{j}(y) \in A\right\}$ (time of first entrance to $\mathrm{A})$, then $k(y)=n(y)+1$. So, $\bar{\tau}_{M}(y)=$ $\tau^{n(y)+1}(y)$. Every orbit of $\bar{\tau}_{M}$ is a subsequence of an orbit of $\tau$.

Claim For all $x \in A$,

$$
\bar{\tau}_{M}(\tau(x))=\tau\left(\tau_{A}(x)\right),
$$

where $\tau_{A}(x)$ is the first return to $A$ map induced by $\tau$.

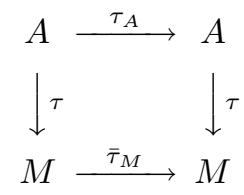

Proof: Suppose $x \in A$. Then

$$
\tau\left(\tau_{A}(x)\right)=\tau^{k+1}(x),
$$

where $k=\min \left\{j \geq 1 \mid \tau^{j}(x) \in A\right\}$. It follows that

$$
\tau\left(\tau_{A}(x)\right)=\tau^{\ell}(x)
$$

where $\ell=k+1=\min \left\{j \geq 1: \tau^{j-1}(x) \in A\right\}$. Since $x=$ $\tau^{1-1}(x) \in A, \ell=\min \left\{j \geq 2: \tau^{j-1}(x) \in A\right\}$. Therefore,

$$
\tau\left(\tau_{A}(x)\right)=\tau^{\ell}(x)=\bar{\tau}_{M}(\tau(x)) .
$$

Proposition 1 For every $N \in \mathbb{N}$ and all $x \in A, \bar{\tau}_{M}^{N}(\tau(x))=$ $\tau\left(\tau_{A}^{N}(x)\right)$.

Proposition 2 If $\bar{\tau}_{M}$ has a periodic orbit, then so does $\tau$. If $\tau$ has a periodic point $x$ such that $\phi(\operatorname{orb}(x))$ is not a singleton, then $\bar{\tau}_{M}$ has a periodic orbit.

Proof: Suppose there exists $y \in M$ and $N \in \mathbb{N}$ such that $\bar{\tau}_{M}^{N}(y)=y$. Then $\tau^{k+N}(y)=y$, where

$$
k=\min \left\{j \geq 2 \mid \tau^{j-1}(y) \in A\right\} .
$$

Conversely, suppose there exists $x \in I$ and $N \geq 2$ such that $\tau^{N}(x)=x$. There is at least one relative $\phi$-maximum in the orbit of $x$; call it $y$. Then $\bar{\tau}_{M}^{j}(y) \in$ $\operatorname{orb}(x)=\left\{x, \tau(x), \ldots, \tau^{N-1}(x)\right\}$, for every $j \in \mathbb{N}$. Therefore, $y$ is periodic under $\bar{\tau}_{M}$.

We define successive $\phi$-maxima map on $\phi(M)$ as follows

Definition 2 (Successive $\phi$-maxima map) The relation

$$
T=\left\{\left(\phi(x), \phi\left(\bar{\tau}_{M}(x)\right)\right) \mid x \in M\right\} \subset \phi(M) \times \phi(M),
$$

is called successive $\phi$-maxima relation of $\tau$. In general $T$ defines a multi-valued map. If $T$ defines a function, it is called the successive $\phi$-maxima map of $\tau$.

A continuous (measurable) function $f: \phi(M) \rightarrow$ $\phi(M)$ is called a continuous (measurable) selection of relation $T$ if for any $x \in \phi(M)$ we have $f(x) \in\{T(x)\}$.

Remark 2 If $T$ defines a map, then it is a factor of $\bar{\tau}_{M}$ : $M \rightarrow M$, as defined by $T(\phi(x))=\phi\left(\bar{\tau}_{M}(x)\right)$, shown in the diagram below. More generally, if $f$ is a selection of $T$, then $f$ is a factor of $\bar{\tau}_{M}$.

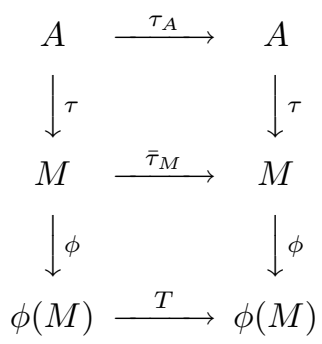




\section{Turbulent trajectories}

In this section we recall the notions of turbulent trajectories [13-15] and horseshoes [17] and prove the main results of the paper, Proposition 6 and Theorem 1 .

Definition 3 The $\omega$-limit set $\omega(x)$ of $x$ is the set of limit points of all convergent subsequences of $\left(\tau^{n}(x)\right)_{n=0}^{\infty}$.

Definition 4 The trajectory $\left\{x, \tau(x), \tau^{2}(x), \ldots\right\}$ is called $\mathrm{S}$ turbulent if $\omega(x)$ is a compact, nonempty set which does not contain periodic points.

Since we consider a compact space $X$, the only real condition in this definition is the absence of the periodic points from $\omega(x)$.

Remark 3

$$
\omega(x)=\bigcap_{n=0}^{\infty} \overline{\tau^{n}\left(\operatorname{orb}_{\tau}(x)\right)}
$$

Now, we will explore the question: Does the existence of a turbulent trajectory for $T$ imply the existence of a turbulent trajectory for the original map $\tau$ ? In general, the answer is no but we will present conditions under which it does hold. We will prove a number of auxiliary results.

First, we will study the relation between turbulent trajectories of $\tau$ and the turbulent trajectories of $\bar{\tau}_{M}$.

Proposition 3 Suppose that $y \in M$ is such that there is a $\tau$-periodic point $z \in \omega_{\tau}(y)$. If $\phi\left(\operatorname{orb}_{\tau}(z)\right)$ has more than one point then there is a $\bar{\tau}_{M}$-periodic point in $\operatorname{orb}(z)$ which is also in $\omega_{\bar{\tau}_{M}}(y)$.

Proof: Let $y \in M$, and suppose that there is a periodic point $z \in \omega_{\tau}(y)$ with period $p$. Suppose that $\phi\left(\operatorname{orb}_{\tau}(z)\right)=\left\{x_{0}, \ldots x_{m-1}\right\} \subset \mathbb{R}$ is not a singleton. Let $0<\varepsilon_{1}<1 / 2$ be chosen so that

$$
B_{\varepsilon_{1}}\left(x_{j}\right) \cap B_{\varepsilon_{1}}\left(x_{k}\right)=\emptyset
$$

for $j \neq k$. Let $0<\delta_{1}$ be chosen so that $\phi\left(B_{\delta_{1}}(t)\right) \subseteq$ $B_{\varepsilon_{1}}(\phi(t))$. Let $0<\gamma_{1}$ be chosen so that $\tau^{i}\left(B_{\gamma_{1}}(t)\right) \subseteq$ $B_{\delta_{1}}\left(\tau^{i}(t)\right)$ for $0 \leq i \leq 2 p$. Then choose $N_{1} \in \mathbb{N}$ so large that $\tau^{N_{1}}(y) \in B_{\gamma_{1}}(z)$. Then by our choice of $\gamma_{1}$ we have

$$
\tau^{N_{1}+j}(y) \in B_{\delta_{1}}\left(\tau^{j}(z)\right)
$$

for $0 \leq j \leq 2 p$. This implies $\phi \circ \tau^{N_{1}+j}(y) \in B_{\varepsilon_{1}}(\phi \circ$ $\left.\tau^{j}(z)\right)$ for $0 \leq j \leq 2 p$. So $\phi \circ \tau^{N_{1}}(y), \phi \circ \tau^{N_{1}+1}(y), \cdots \phi \circ$ $\tau^{N_{1}+p}(y)$ visit each open set $B_{\varepsilon_{1}}\left(x_{0}\right), B_{\varepsilon_{1}}\left(x_{1}\right), \ldots B_{\varepsilon_{1}}\left(x_{m}\right)$ and return to the first. This implies that there is some $0 \leq k \leq p$ with

$$
\phi \circ \tau^{N_{1}+k}(y) \leq \phi \circ \tau^{N_{1}+k+1}(y) \geq \phi \circ \tau^{N_{1}+k+2}(y),
$$

i.e., $\tau^{N_{1}+k+1}(y) \in M$ and there is some $\ell_{1}$ with $\phi \circ$ $\tau^{N_{1}+k+1}(y) \in B_{\varepsilon_{1}}\left(x_{\ell_{1}}\right)$.

Continue the argument to get a point $x_{r}=\phi \circ$ $\tau^{s}(z) \in \phi\left(\operatorname{orb}_{\tau}(z)\right)$ and a subsequence $N_{i_{j}}+k_{i_{j}}+1$ such that

$$
\tau^{N_{i_{j}}+k_{i_{j}}+1}(y) \in M
$$

and

$$
\begin{aligned}
& \tau^{N_{i_{j}}+k_{i_{j}}+1}(y) \in B_{\gamma_{j}}\left(\tau^{s}(z)\right) . \\
& \phi \circ \tau^{N_{i_{j}}+k_{i_{j}}+1}(y) \in B_{\varepsilon_{j}}\left(x_{r}\right)
\end{aligned}
$$

and

$$
\phi \circ \tau^{N_{i_{j}}+k_{i_{j}}+1}(y) \rightarrow x_{r} .
$$

But more importantly by ensuring $\gamma_{j} \rightarrow 0$ we have

$$
\tau^{N_{i_{j}}+k_{i_{j}}+1}(y) \rightarrow \tau^{s}(z)
$$

Thus there is some $0 \leq s<p$ with $\tau^{s}(z) \in \omega_{\bar{\tau}_{M}}(y)$.

Now, we will study the relation between turbulent trajectories of $\bar{\tau}_{M}$ and the turbulent trajectories of $T$. We say that a sequence $\left(t_{0}, t_{1}, t_{2}, \ldots, t_{n}, t_{n+1}, \ldots\right)$ forms a trajectory of $T$ if we have $t_{n+1} \in T\left(t_{n}\right)$ for all $n=$ $0,1,2, \ldots$

Proposition 4 If $\omega_{\bar{\tau}_{M}}(x)$ contains a periodic point, then the set $\omega_{T}(\phi(x))$ contains a periodic point.

Proof: First, we will prove that if $\bar{\tau}_{M}$ has a periodic trajectory $\left\{y_{i}=\bar{\tau}_{M}^{i}(y)\right\}_{i=0}^{p}$, then points $\left\{\phi\left(y_{i}\right)\right\}_{i=0}^{p}$ form a periodic trajectory of $T$. This holds since for any $0 \leq i \leq p$ we have $\phi\left(\bar{\tau}_{M}\left(y_{i}\right)\right)=\phi\left(y_{i+1}\right) \in T\left(\phi\left(y_{i}\right)\right)$, with the understanding that $p+1=0$.

Assume that $\omega_{\bar{\tau}_{M}}(x)$ contains a periodic point. The trajectory $\left\{\bar{\tau}_{M}^{i}(x)\right\}_{i=0}^{\infty}$ is projected by $\phi$ into the set $\left\{T^{i}(\phi(x))\right\}_{i=0}^{\infty}$ since for any $i \geq 0$ we have $\phi\left(\bar{\tau}_{M}^{i+1}(x)\right) \in$ $T\left(\phi\left(\bar{\tau}_{M}^{i}(x)\right)\right)$. Thus, since $\phi$ is continuous we have

$$
\phi\left(\omega_{\bar{\tau}_{M}}(x)\right) \subset \omega_{T}(\phi(x)) .
$$

By the first part of this proof, $\omega_{T}(\phi(x))$ contains a period point.

Proposition 3 cannot be improved, i.e., the assumption $\phi(\operatorname{orb}(z))$ is not a singleton cannot be omitted. This is shown by a counterexample in the next section.

We will use Proposition 3 to show that if consecutive maxima map $T$ (possibly multi-valued) has a horseshoe, then the original map $\tau$ has an "almost turbulent trajectory", i.e., a trajectory whose $\omega$-set is infinite and does not contain a periodic trajectory with period larger than 1. It may contain a fixed point which has to be checked separately.

We will assume that $\tau$ has only isolated periodic points. Such an assumption is often made and a map $\tau$ generic in any reasonable sense satisfies it. Then, $\tau$ has a countable number of periodic points. We will 
prove that a generic $\phi$ sends elements of any periodic trajectory with period $k>1$ onto $k$ distinct values. We say that $\phi \in C^{0}(X, \mathbb{R})$ separates periodic orbit $O=\left\{z_{1}, z_{2}, \ldots, z_{k}\right\}, k>1$, if $\phi\left(z_{i}\right) \neq \phi\left(z_{j}\right)$, for at least one pair $1 \leq i<j \leq k$.

Lemma 1 Let $X$ be a normal infinite space without isolated points. The set of functions $\phi \in C^{0}(X, \mathbb{R})$ which separate all periodic orbits of periods larger than 1 is generic, i.e., of second Baire's category in $C^{0}(X, \mathbb{R})$.

Proof: Let $O=\left\{z_{1}, z_{2}, \ldots, z_{k}\right\}, k>1$, be a periodic trajectory of $\tau$. The set of functions $\phi$ such that $\phi\left(z_{i}\right)=$ $\phi\left(z_{j}\right)$ is closed and nowhere dense in $C^{0}(X, \mathbb{R})$, for any $z_{i} \neq z_{j}$. Thus, its complement is open and dense. Then, the set of $\phi$ 's which separate $O$ is open and dense as a finite intersection of such sets. Then, the set of $\phi$ 's which separate all periodic orbits of $\tau$ is of second Baire's category in $C^{0}(X, \mathbb{R})$.

Definition 5 We say that the map $T=T_{\phi}$ defined using the function $\phi$ has a "horseshoe" when there exist mutually disjoint compact subsets $J_{1}, J_{2}, \ldots, J_{k} \subset$ $\phi(M)$ and a selection $T_{1}$ of $T_{\phi}$ such that, for any $1 \leq$ $i \leq k$, we have

$$
T_{1}\left(\operatorname{Int} J_{i}\right) \supset J_{1} \cup J_{2} \cup \ldots \cup J_{k},
$$

where Int $J$ is the interior of $J$.

Remark 4 It seems that chaoticity of a system is strongly related to the existence of horseshoes. In particular, in dimension one, a continuous piecewise monotonic map with positive entropy has horseshoes [17].

Definition 6 We say that the multi-valued map $T=$ $T_{\phi}$, defined using function $\phi$, has a "generalized horseshoe" when there exist mutually disjoint compact subsets $J_{1}, J_{2}, \ldots, J_{k} \subset \phi(M)$ and a selection $T_{1}$ of $T_{\phi}$ such that, for any $1 \leq i \leq k$, we have

$$
T_{1}\left(\operatorname{Int} J_{i}\right) \supset J_{j}
$$

for any $1 \leq i, j \leq k$ such that $a_{i j}=1$ in a 0,1 mixing matrix $A=\left(a_{i j}\right)_{1 \leq i, j \leq k}$.

Lemma 2 If $T_{\phi}$ has a horseshoe or a generalized horseshoe $J_{1}, J_{2}, \ldots, J_{k}$, then there exists a neighbourhood of $\mathcal{U} \subset C^{0}(X, \mathbb{R})$ of $\phi$ such that $J_{1}, J_{2}, \ldots, J_{k}$ form a horseshoe for any $T_{\psi}$ with $\psi \in \mathcal{U}$.

Proof: The condition $T_{1}\left(\operatorname{Int} J_{i}\right) \supset J_{1} \cup J_{2} \cup \ldots \cup J_{k}$ is preserved when we change $\phi$ sufficiently little in $C^{0}(X, \mathbb{R})$.

Definition 7 A trajectory whose $\omega$-set is infinite and does not contain a periodic trajectory with period larger than 1 is called an almost turbulent trajectory.
Proposition 5 If $T$ has a horseshoe or a generalized horseshoe, then $\tau$ has an almost turbulent trajectory.

Proof: Let $T=T \phi$. By Lemma 1, arbitrarily close to $\phi$, there is a $\psi$ which separates all periodic orbits of $\tau$. If we take $\psi$ sufficiently close to $\phi$, by Lemma $2 \psi$ also has a horseshoe. The existence of a horseshoe implies the existence of a turbulent trajectory [15]. Then, by Proposition $4, \bar{\tau}_{M}$ defined using $\psi$ has a turbulent trajectory, and by Proposition 3, $\tau$ has an almost turbulent trajectory. The only thing we cannot control is the possibility that this trajectory has a fixed point in its $\omega$-set.

Proposition 6 If $T$ has a horseshoe or a generalized horseshoe $J_{1}, J_{2}, \ldots, J_{k}$, such that $\cup_{i=1}^{k} J_{i}$ is disjoint from $\phi(\mathcal{U})$, where $\mathcal{U}$ is some neighbourhood of fixed points of $\tau$, then $\tau$ has a turbulent trajectory.

Proof: By [15] the turbulent set of $T$ is contained in the union of sets forming a horseshoe. Thus, the turbulent trajectory of $\tau$ obtained in Proposition 5 is disjoint from $\mathcal{U}$ and cannot have a fixed point in its $\omega$-set.

Using the results of [13] we obtain the following:

Theorem 1 If $\tau$ is continuous and the successive maxima map $T$ has a horseshoe or a generalized horseshoe disjoint from $\phi$ image of a neighbourhood of fixed points of $\tau$, then the transformation $\tau$ has a continuous ergodic invariant measure.

\section{Can Proposition 3 be improved ?}

The answer to the question above is no. The assumption $\phi(\operatorname{orb}(z))$ is not a singleton cannot be omitted in general. A counterexample follows.

Example 1 Let $C \subset[0,1]$ be the usual Cantor middle third set and define $T: C \rightarrow C$ to be the tent map given by

$T(x)=\left\{\begin{array}{cl}3 x & : x \leq 1 / 3 \\ 3(1-x) & : x \geq 2 / 3\end{array}\right.$

Let $c \in C$ be a point for which $\omega_{T}(c)=C$.

Now, let $X=C \times S$ where $S$ is the circle with representation $\mathbb{R} / \mathbb{Z}$. Let $\alpha \in[0,1]$ be irrational, and define $\tau: X \rightarrow X$ by

$\tau(x, \theta)=\left\{\begin{array}{cl}(T(x), \theta) & : x \in[0,1 / 9] \cup[8 / 9,1] \\ (T(x), \theta+\alpha) & : x \in[2 / 9,1 / 3] \cup[2 / 3,7 / 9]\end{array}\right.$

That is, $\tau$ maps $(x, \theta)$ to a point with first coordinate $T(x)$ and with second coordinate unchanged if $T(x) \leq$ $1 / 3$. If $T(x) \geq 2 / 3$, then $\tau$ also rotates the point by the irrational angle $\alpha$. 
Now, define $\phi: X \rightarrow \mathbb{R}$ by $\phi(x, \theta)=x$. Since $\phi$ depends only on $x$,

$$
\begin{aligned}
A & =\left\{x \in X: \phi(x, \theta) \leq \phi(\tau(x, \theta)) \geq \phi\left(\tau^{2}(x, \theta)\right)\right\} \\
& =\left\{x \in X: x \leq T(x) \geq T^{2}(x)\right\} \times S .
\end{aligned}
$$

Checking the inequalities under iteration of $T$, we see that

$A=(\{0\} \cup(([1 / 4,1 / 3] \cup[2 / 3,3 / 4]) \cap C)) \times S$

and so

$$
M=(\{0\} \cup([3 / 4,1] \cap C)) \times S
$$

Let $y=(c, 0) \in X$ where $c \in C$ was chosen to have $\omega_{T}(c)=C$. Observe that, for all $\epsilon>0$ and $N \in \mathbb{N}$, there exists $n \geq N$ such that $0 \leq T^{n}(c)<\epsilon$, and thus there exists $x \in\{0\} \times S$ with $x \in \omega_{\tau}(y)$. Since $T$ is the identity map on $\{0\} \times S, x$ is fixed and hence periodic. Thus, $\omega_{\tau}(y)$ contains a periodic point.

However, notice that for all $n \in \mathbb{N}, T^{n}(c) \neq 0$, so that $\tau^{n}(y) \notin\{0\} \times S$. In particular, $\bar{\tau}_{M}^{n}(y) \in[3 / 4,1] \times S$ for all $n \in \mathbb{N}$. Thus $\omega_{\bar{\tau}_{M}}(y) \subseteq[3 / 4,1] \times S$. For all $(x, \theta) \in$ $[3 / 4,1] \times S$, it is apparent that $\tau^{n}(x, \theta)=\left(T^{n}(x), \theta+\right.$ $k \alpha)$ for some $0 \leq k \leq n$. However, for $\tau^{n}(x, \theta)$ to be an element of $M$, it must be the case that $k>0$. Thus, for all $n \in \mathbb{N}, \bar{\tau}_{M}^{n}(x, \theta)=\left(T^{k}(x), \theta+j \alpha\right)$ for some $k, j \geq$ $n$. In particular, $\bar{\tau}_{M}$ rotates the angle by an integer multiple of the irrational angle $\alpha$, and hence for all $n \in$ $\mathbb{N}, \bar{\tau}_{M}^{n}(x, \theta) \neq(x, \theta)$. Thus, $(x, \theta)$ is not periodic and $\omega_{\bar{\tau}_{M}}(y)$ contains no periodic points.

Remark 5 The map $\tau$ above can be altered to use a rational rotation rather than a zero rotation on $[0,1 / 9] \cup$ $[8 / 9,1] \times S$, which will yield $\omega_{\tau}(y)$ having non-fixed periodic points.

\section{Invariant measure for $\bar{\tau}_{M}$ implies invariant measure for $\tau$}

Let us define $\tau_{M, A}: M \rightarrow A$ to be the first visit map to the set $A$ :

$$
\tau_{M, A}(x)=\tau^{k}(x),
$$

where, $\tau^{k}(x) \in A, k>0, \tau^{j}(x) \notin A, j=1,2, \ldots, k-1$. Then, we have

$$
\bar{\tau}_{M}=\tau \circ \tau_{M, A} \text { and } \tau_{A}=\tau_{M, A} \circ \tau .
$$

Let us assume that $\bar{\tau}_{M}$ admits an invariant measure $\mu$, i.e., for any measurable subset $B$ of $M$ we have

$$
\mu\left(\bar{\tau}_{M}^{-1}(B)\right)=\mu(B) .
$$

Let us define a measure $\nu$ on $A$ as follows:

$$
\nu(Z)=\mu\left(\tau_{M, A}^{-1}(Z)\right) .
$$

Then, we have

$$
\begin{aligned}
\nu\left(\tau_{A}^{-1}(Z)\right)= & \mu\left(\tau_{M, A}^{-1}\left(\tau_{A}^{-1}(Z)\right)\right)=\mu\left(\tau_{M, A}^{-1}\left(\tau^{-1}\left(\tau_{M, A}^{-1}(Z)\right)\right)\right) \\
& =\mu\left(\bar{\tau}_{M}^{-1}\left(\tau_{M, A}^{-1}(Z)\right)\right)=\mu\left(\tau_{M, A}^{-1}(Z)\right)=\nu(Z) .
\end{aligned}
$$

We proved that the first return map $\tau_{A}$ has an invariant measure. Then, it follows that $\tau$ also has an invariant measure. By the nature of the construction, if $\mu$ is absolutely continuous and $\tau$ is non singular then the $\tau$-invariant measure is also absolutely continuous.

\section{Conclusion:}

Let $\tau$ be a continuous map on a compact metric space $X$. Given a continuous function $\phi: X \rightarrow \mathbb{R}$ we consider a 1-dimensional map $T$ (possibly multi-valued) which sends a local $\phi$-maximum on the $\tau$ trajectory to the next one. We call this the consecutive maxima map. In our main result we prove that if $T$ has a horseshoe disjoint from the projections of fixed points, then $\tau$ is in some sense chaotic, i.e., it has a turbulent trajectory and thus a continuous invariant measure. A number of examples are presented.

\section{Examples}

6.1 Maxima map for a discretization of the Lorenz system

Edward Lorenz [16] introduced a mathematical model for atmospheric dynamics, which is a system of three ordinary differential equations:

$\dot{x}=\sigma(y-x)$,

$\dot{y}=x(\rho-z)-y$,

$\dot{z}=x y-\beta z$,

where $\sigma, \rho$ and $\beta$ are system parameters. Lorenz used $\sigma=10, \rho=28$ and $\beta=8 / 3$. This system exhibits chaotic behaviors and a strange attractor exists [23].

Instead of considering the above continuous system, we introduce a discrete Lorenz system. Letting $h$ be the step size, $n \in \mathbb{N}$, we rewrite the above system as follows:

$\frac{x((n+1) h)-x(n h)}{h}=\sigma(y(n h)-x(n h))$,
$\frac{y((n+1) h)-y(n h)}{h}=x(n h)(\rho-z(n h))-y(n h)$,

$\frac{z((n+1) h)-z(n h)}{h}=x(n h) y(n h)-\beta z(n h)$.

This simplifies to

$x((n+1) h)=h \sigma y(n h)+(1-h x(n h))$,

$y((n+1) h)=h x(n h)(\rho-z(n h))+(1-h) y(n h)$,

$z((n+1) h)=h x(n h) y(n h)+(1-h \beta) z(n h)$. 
We define the discrete Lorenz system $L: \mathbb{R}^{3} \rightarrow \mathbb{R}^{3}$ as

$L\left(\begin{array}{l}x \\ y \\ z\end{array}\right)=\left(\begin{array}{c}h \sigma y+(1-h \sigma) x \\ h x(\rho-z)+(1-h) y \\ h x y+(1-h \beta) z\end{array}\right)$.

An example of a trajectory of this discrete system is shown in Figure 1.

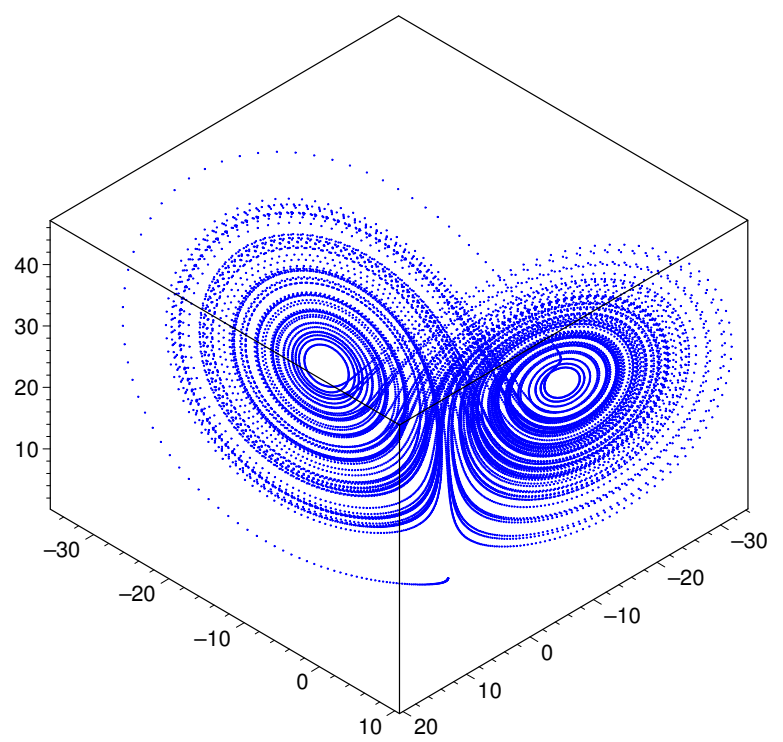

Fig. 1 A plot of the orbit starting at $(0.1,-0.3,1.7)$ using $\sigma=10, \rho=28, \beta=8 / 3$ and $h=0.005$ with 20000 iterations for system (1).

For a given function $\phi: \mathbb{R}^{3} \rightarrow \mathbb{R}$ our successive $\phi$ maxima map is defined by the relation

$T=\left\{\left(\phi(x), \phi\left(\bar{\tau}_{M}(x)\right)\right) \mid x \in M\right\}$.

Now, we list some examples of successive maxima maps associated with different functions $\phi$.

Case(I): Let $\phi: \mathbb{R}^{3} \rightarrow \mathbb{R}$ be defined as $\phi(x, y, z)=$ $z$, and let $h=0.005$. The set $M$ is shown in Figure 3, and the corresponding successive maxima map is shown in Figure 4.

We fitted a curve to data plotted in Figure 4. It turns out that the fitting map is piecewise expanding, with two onto branches. Thus, it is topologically conjugated to the standard tent map. The formula for the first branch is

$$
\begin{aligned}
T(x)= & -754.1523850+71.23641413 x-2.182157170 x^{2} \\
& +.02266171341 x^{3}, \quad 28 \leq x \leq 48.1 .
\end{aligned}
$$

Below we provide an example of a generalized horseshoe for the standard tent map. Let $\varepsilon$ be a small positive number $($ say $<0.01)$. The intervals $I_{1}=[2 / 12-$ $\varepsilon, 3 / 12+\varepsilon], I_{2}=[4 / 12-\varepsilon, 6 / 12+\varepsilon]$ and $I_{3}=[9 / 12-$ $\varepsilon, 11 / 12+\varepsilon]$ form a horseshoe (Figure 2 ) with a matrix

$A=\left[\begin{array}{lll}0 & 1 & 0 \\ 0 & 0 & 1 \\ 1 & 1 & 0\end{array}\right]$.

$A$ is mixing and its largest eigenvalue is 1.3247 .

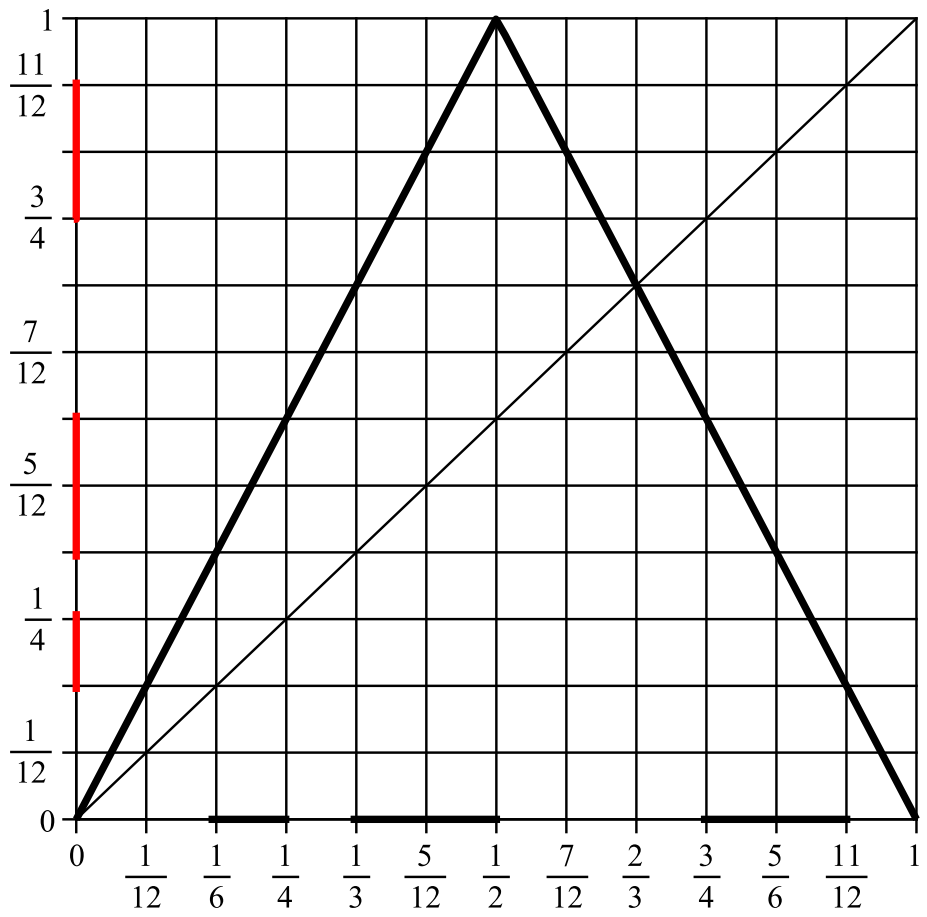

Fig. 2 Intervals forming a generalized horseshoe for tent map.

Case(II): Let us consider $\phi(x, y, z)=\epsilon x+\xi y+z$, for $(x, y, z) \in \mathbb{R}^{3}$ and $h=0.005, \epsilon=0.001, \xi=0.001$. The set $M$ is shown in Figure 5, and the corresponding successive maxima map is shown in Figure 6.

We fitted a curve to data plotted

\subsection{Maxima map for one humped map $\tau$}

Let $I=[0,1] . \tau$ is a one humped map of $[0,1]$, i.e., $\tau(0)=\tau(1)=0, \tau(c)=1, \tau$ is increasing on $[0, c]$ and decreasing on $[c, 1]$.

$\tau$ has two fixed points 0 and $a$. For any $x \in[0, a]$ we have $\tau(x) \geq x$ and for any $x \in[a, 1]$ we have $\tau(x) \leq x$. Thus, no point in $[0, a]$ is in $M$ and $(a, 1]=M$. Let $b$ be the other preimage of $a$, i.e., $\tau(b)=a$ and $b<a$. Then, $\tau^{-1}([a, 1])=[b, a]$ and the only way to fall into $M$ is go through $[b, a]$. This shows that in the case of a 


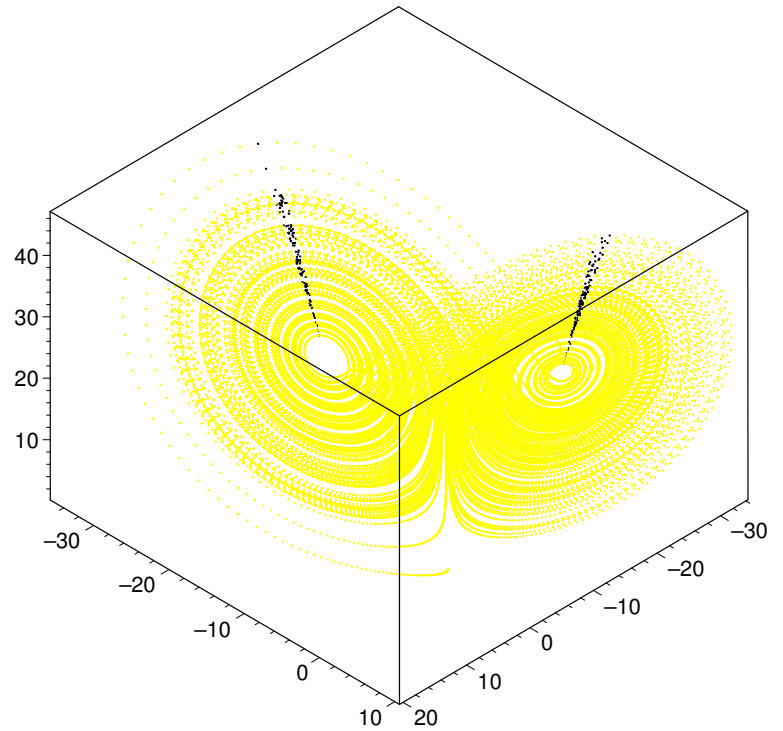

Fig. 3 A plot of the orbit starting at $(0.1,-0.3,1.7)$ for system $(1)$, the set $M$ is shown in black using $\phi(x, y, z)=z$.

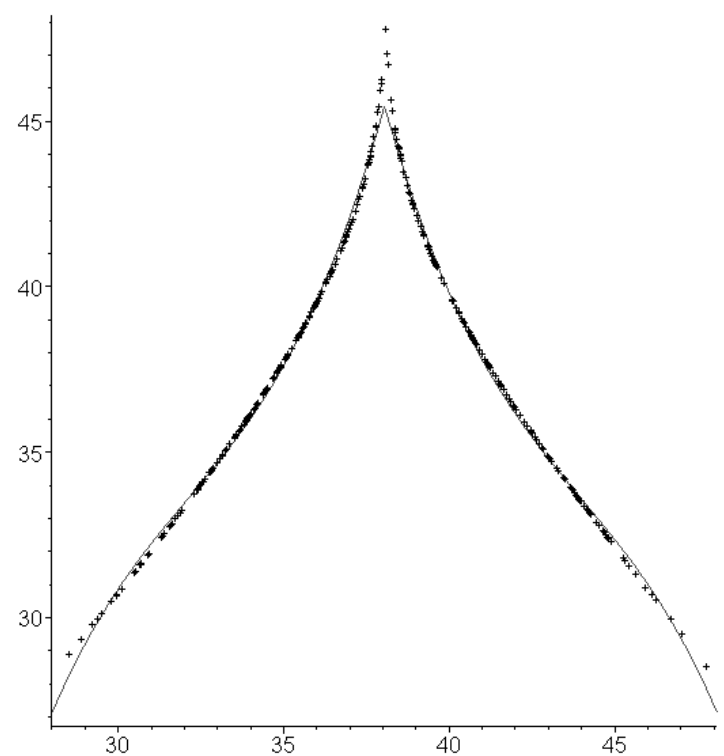

Fig. 4 The successive maxima map for the set $M$ shown in Figure 3 and $\phi(x, y, z)=z, h=0.005$. Thin line shows the fitted curve.

one humped map, we have

$$
\bar{\tau}_{M}=\tau_{M}=\tau_{[a, 1]},
$$

where $\tau_{[a, 1]}$ is the first return map for the subinterval $[a, 1]$. Note that in this case $\tau_{M}=\tau \circ \tau_{A}$, where $A=$ $[b, a]$

\subsection{Maxima map for second iterate of tent map $\tau$}

The set

$$
A=[0.1,1 / 6] \cup[0.2,0.3] \cup[1 / 3,0.4] \cup[0.7,0.8],
$$

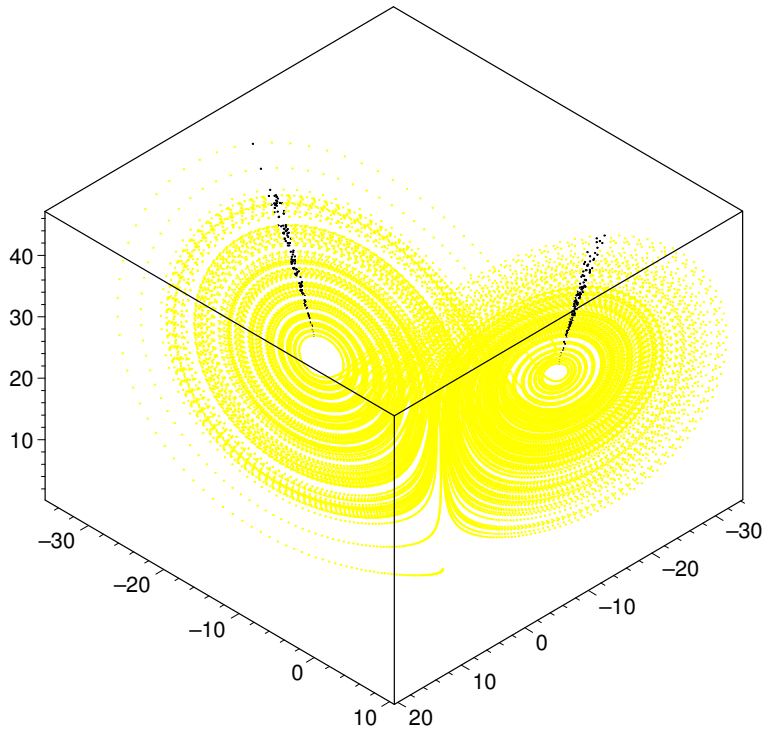

Fig. 5 A plot of the orbit starting at $(0.1,-0.3,1.7)$ for system (1), the set $M$ is shown in black using $\phi(x, y, z)=\epsilon x+\xi y+z$.

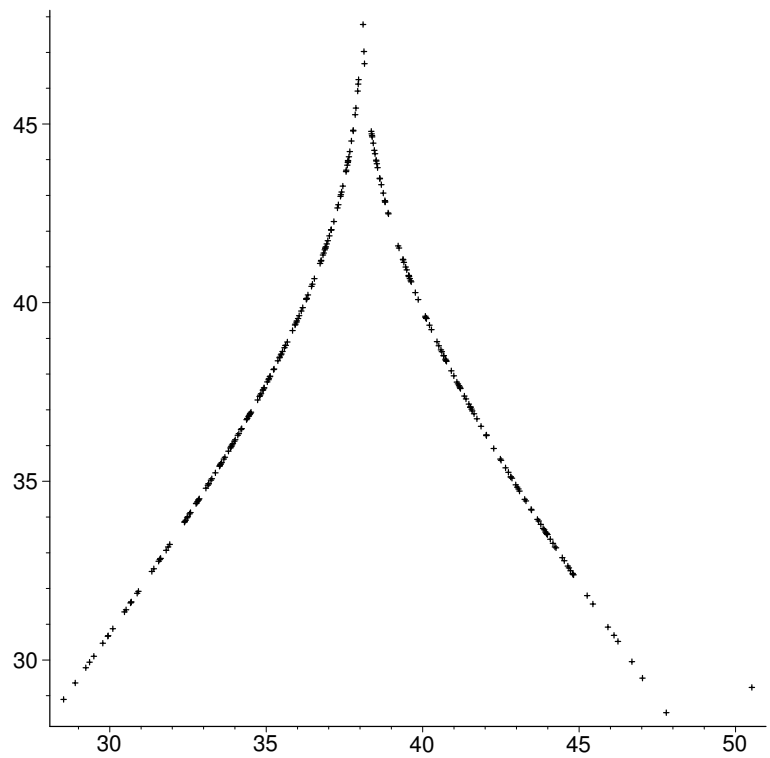

Fig. 6 The successive maxima map for the set $M$ shown in Figure 5 and $\phi(x, y, z)=\epsilon x+\xi y+z, h=0.005$

and set

$$
M=[0.4,2 / 3] \cup[0.8,1] .
$$

In this case the map $\bar{\tau}_{M}$ is not the first return map to the set $M$.

6.4 Consecutive maxima relation for rotation of a circle

Let $\tau: S^{1} \rightarrow S^{1}$ be a rotation through angle $\alpha$. We assume that $\alpha<2 \pi / 3$ and is not of the form $2 \pi / n$ for a natural $n$. Let $\phi: S^{1} \rightarrow \mathbb{R}$ be the function $\phi(t)=$ $\cos (t)$, i. e., the value of the $x$ coordinate of the point 


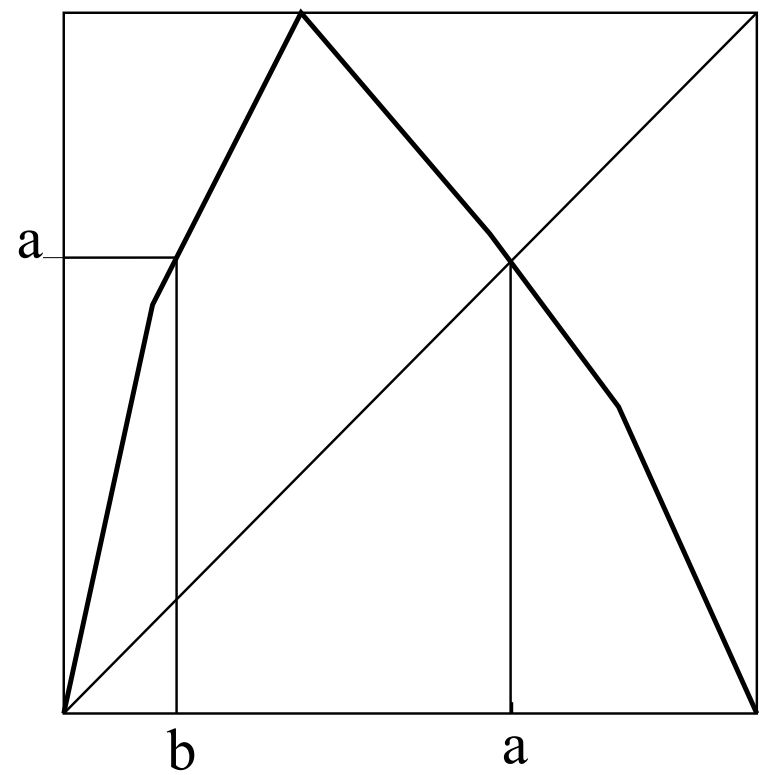

Fig. 7 Caption for first_return

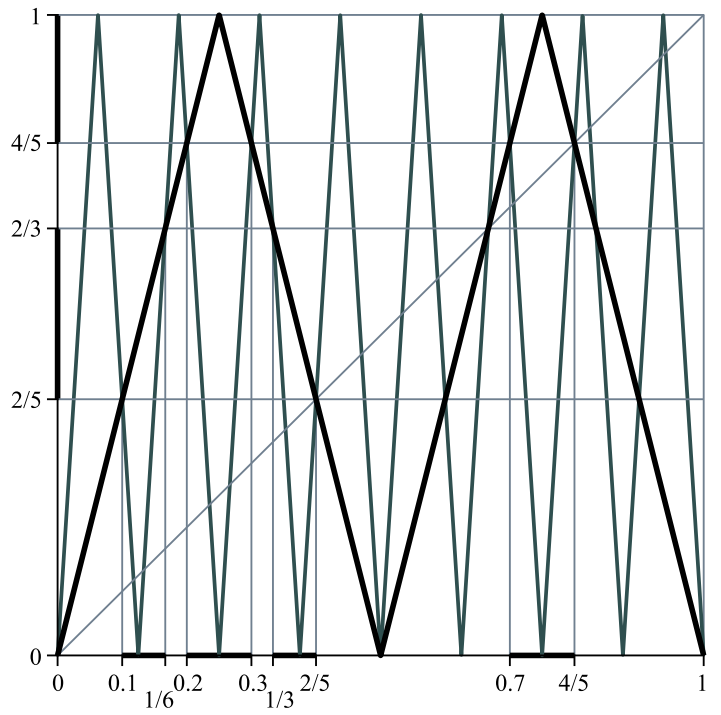

Fig. 8 Sets $A$ (on x-axis) and $M$ (on y-axis) for second iterate of the tent map.

$(x, y)=(\cos (t), \sin (t)) \in S^{1}, t \in[0,2 \pi)$. We will identify points on $S^{1}$ with their arguments $t$. The arguments are considered modulo $2 \pi$. It is easy to see that

$$
\begin{aligned}
A & =\left\{t \in[0,2 \pi): \phi\left(\tau^{2}(t)\right) \leq \phi(\tau(t)) \geq \phi(t)\right\} \\
& =[-\alpha / 2-\alpha,-\alpha / 2]
\end{aligned}
$$

and

$$
M=\tau(A)=[-\alpha / 2, \alpha / 2] .
$$

Since $\tau$ is one-to-one, $\bar{\tau}_{M}=\tau \circ \tau_{A}=\tau_{M}$, the first return to $M$ map. Let $t \in[-\alpha / 2, \alpha / 2]=M$. The time $n(t)$ of the first return to $M$ can be found as the smallest integer $n$ such that

$$
-\alpha / 2+2 \pi \leq n \alpha+t \leq \alpha / 2+2 \pi,
$$

or

$$
n \in[(2 \pi-t) / \alpha-1 / 2,(2 \pi-t) / \alpha+1 / 2] .
$$

Thus,

$$
n(t)=E((2 \pi-t) / \alpha-1 / 2))+1
$$

except for one $t=2 \pi-n \alpha-\alpha / 2$. Thus,

$\left.\tau_{M}(t)=t+n(t) \alpha=t+(E((2 \pi-t) / \alpha-1 / 2))+1\right) \alpha$.

Thus, the consecutive maxima two-valued map $T$ is defined as

$$
\begin{aligned}
x \rightarrow & \arccos (x)+n(\arccos (x)) \alpha, \\
& \quad-\arccos (x)+n(-\arccos (x)) \alpha\} \\
= & \{\arccos (x)+(E((2 \pi-\arccos (x)) / \alpha-1 / 2))+1) \alpha, \\
& \quad-\arccos (x)+(E((2 \pi+\arccos (x)) / \alpha-1 / 2))+1) \alpha\},
\end{aligned}
$$

for $x \in \phi(M)=[\cos (\alpha / 2), 1]$.

\section{Example 2}

For $\alpha=2.15722723$ we have $\cos (\alpha / 2)=0.4725506413$ and the graph of $T$ is shown in Figure 9 (a).

More interesting is the graph of $T^{3}$ shown in Figure 9 (b). It contains two onto "branches", drawn in black. It shows that the intervals $J_{1}=\left[p_{1}, p_{2}\right], J_{2}=\left[p_{2}, 1\right]$ $\left(p_{1}=\cos (\alpha / 2), p_{2} \approx 0.8712149\right.$ is the solution of the equation $\left.T^{3}(x)=p_{1}\right)$ form a kind of "horseshoe" for $T^{3}$, i.e., $T^{3}\left(J_{i}\right) \supset J_{1} \cup J_{2}, i=1,2$. This is not a horseshoe satisfying our definition since it is not a horseshoe for any selection of $T^{3}$.

The results of numerical simulation suggest that two-valued map $T$ has an absolutely continuous invariant measure.

6.5 Relation $T$ for rotation of a circle through angle $\alpha=\pi / 4$

Let $\tau: S^{1} \rightarrow S^{1}$ be the rotation through angle $\alpha=\pi / 4$. Let $\phi: S^{1} \rightarrow \mathbb{R}$ be the function $\phi(t)=\cos (t)$, i.e., the value of the $x$ coordinate of the point $(x, y)=$ $(\cos (t), \sin (t)) \in S^{1}, t \in[0,2 \pi)$. We will identify points on $S^{1}$ with their arguments $t$. The arguments are considered modulo $2 \pi$. The two valued map $T:[-1,1] \rightarrow$ $[-1,1]$ is defined by the relation $T(\phi(t))=\phi(\tau(t))$, $t \in[0,2 \pi)$, or

$$
\begin{aligned}
x & \rightarrow\left\{T_{1}(x), T_{2}(x)\right\} \\
& =\{\cos (\arccos (x)+\pi / 4), \cos (-\arccos (x)+\pi / 4)\},
\end{aligned}
$$

for $x \in \phi\left(S^{1}\right)=[-1,1]$. 


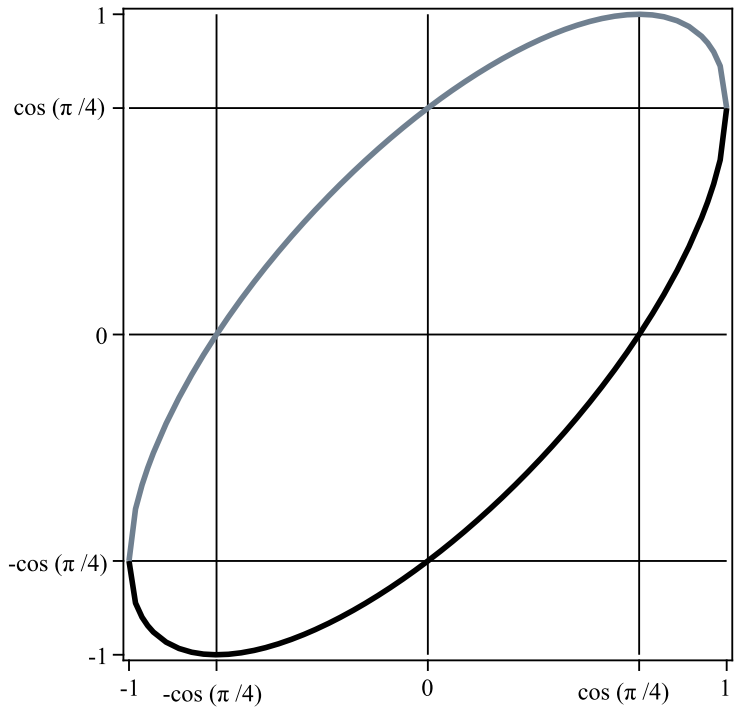

Fig. 10 Graph of relation $T$ for rotation through angle $\alpha=$ $\pi / 4, T_{1}$ in black, $T_{2}$ in gray.

The graph of $T$ is shown in Figure ??.

We consider partition of $[-1,1]$ by points $-1,-\cos (\pi / 4)$ $=-\sqrt{2} / 2,0, \sqrt{2} / 2,1$ into intervals $J_{1}=[-1,-\sqrt{2} / 2]$, $J_{2}=[-\sqrt{2} / 2,0], J_{3}=[0, \sqrt{2} / 2], J_{4}=[\sqrt{2} / 2,1]$. The incidence matrix of $T$ with this partition is

$$
M=\left[\begin{array}{llll}
1 & 1 & 0 & 0 \\
1 & 0 & 1 & 0 \\
0 & 1 & 0 & 1 \\
0 & 0 & 1 & 1
\end{array}\right] .
$$

The maximal eigenvalue of $M$ is 2 , which shows that the entropy of $T$ is at least $\ln (2)$. (See Section ??.) At the same time each trajectory of $\tau$ (and of $T$ ) consists of only a finite number of points.

We used topological entropy for relations as defined in $[10,11,19,7,21]$. Let $T$ be a relation on a compact metric space $(X, d)$. A sequence $\left(x_{0}, x_{1}, x_{2}, \ldots, x_{k-1}\right) \in$ $X^{k}$ is called a trajectory of $x_{0}$ if $x_{i+1} \in T\left(x_{i}\right)$, for $i=0,1, \ldots, k-2$. We define the distance $d_{k}$ between trajectories of length $k$ as follows

$$
\begin{aligned}
& d_{k}\left(\left(x_{0}, x_{1}, x_{2}, \ldots, x_{k-1}\right),\left(y_{0}, y_{1}, y_{2}, \ldots, y_{k-1}\right)\right) \\
= & \max _{0 \leq i \leq k-1} d\left(x_{i}, y_{i}\right) .
\end{aligned}
$$

The set $\mathcal{A}$ of trajectories of length $k$ is called $(\varepsilon, k)$ separated if for any two trajectories $\bar{x}, \bar{y} \in \mathcal{A}$ we have $d_{k}(\bar{x}, \bar{y})>\varepsilon$. Since $X$ is compact, such a set is finite. Let us define $S(\varepsilon, k)$ as the supremum of cardinalities of all $(\varepsilon, k)$ separated sets. The topological entropy of $T$ is defined as

$$
h(T)=\lim _{\varepsilon \rightarrow 0} \limsup _{k \rightarrow \infty} \frac{1}{k} \ln S(\varepsilon, k) .
$$

Acknowledgements The authors are very grateful to anonymous reviewers for comments which helped to improve the presentation of the paper.

\section{References}

1. Alligood, Kathleen T., Sauer, Tim D. and Yorke, James A., Chaos. An introduction to dynamical systems, Textbooks in Mathematical Sciences. Springer-Verlag, New York, (1997).

2. Banks, J., Dragan, V., Jones, A., Chaos, A Mathematical Introduction, Cambridge University Press, (2003).

3. Boyarsky, Abraham; Góra, Paweł; Laws of chaos. Invariant measures and dynamical systems in one dimension, Probability and its Applications. Birkhäuser Boston, Inc., Boston, MA, (1997).

4. Boyarsky, Abraham, Góra, Paweł, Lioubimov, Vadim, On the existence of ergodic continuous invariant measures for folding transformations, Ergodic Theory Dynam. Systems 20, no. 1, 47-53, (2000).

5. Brin, Michael and Stuck, Garrett, Introduction to dynamical systems, Cambridge University Press, Cambridge, (2002).

6. Brown, James R., Ergodic Theory and Topological Dynamics, Academic Press, N.Y, (1976).

7. Cheng, Wen-Chiao(RC-CHNG); Newhouse, Sheldon E., Pre-image entropy, Ergodic Theory Dynam. Systems 25, no. 4, 1091-1113, (2005).

8. Manfred Denker, Christian Grillenberger and Karl Sigmund, Ergodic Theory on Compact Spaces, Lect. Notes in Math. Vol. 527, Springer-Verlag, Berlin, Heidelberg, New York (1976), DOI: 10.1007/BFb0082364.

9. Devaney, Robert L., A first course in chaotic dynamical systems. Theory and experiment. Addison-Wesley Studies in Nonlinearity. Addison-Wesley Publishing Company, Advanced Book Program, Reading, MA, (1992).

10. Langevin, Rémi; Przytycki, Félix, Entropie de l'image inverse d'une application. [Entropy of the inverse image of a mapping], Bull. Soc. Math. France 120, no. 2, 237250, (1992).

11. Langevin, Rémi; Walczak, Paweł, Entropie dune dynamique. [Entropy of a dynamic], C. R. Acad. Sci. Paris Sér. I Math. 312, no. 1, 141-144, (1991).

12. Lasota, Andrzej, Mackey, Michael C., Chaos, fractals, and noise. Stochastic aspects of dynamics, Second edition. Applied Mathematical Sciences, 97. SpringerVerlag, New York, (1994).

13. Lasota, Andrzej; Yorke, James A., On the existence of invariant measures for transformations with strictly turbulent trajectories, Bull. Acad. Polon. Sci. Sér. Sci. Math. Astronom. Phys. 25, no. 3, 233-238, (1977).

14. Lasota, A., Pianigiani, G., Invariant measures on topological spaces, Boll. Un. Mat. Ital. B (5) 14, no. 2, 592603, (1977).

15. Lasota, A., Yorke, James A., On the existence of invariant measures for transformations with strictly turbulent trajectories, Bull. Acad. Polon. Sci. Sr. Sci. Math. Astronom. Phys. 25, no. 3, 233-238, (1977).

16. E.N. Lorenz, Deterministic Non-periodic Flow, J. Atmos. Sci. 20, 130-141, (1963).

17. Misiurewicz, Michal, Horseshoes for mappings of the interval, Bull. Acad. Polon. Sci. Sr. Sci. Math. 27, no. 2, 167-169, (1979).

18. Misiurewicz, Michal, Absolutely continuous measures for certain maps of an interval, Inst. Hautes Études Sci. Publ. Math. No. 53, 17-51, (1981). 
19. Z. Nitecki and F. Przytycki, Preimage entropy for mappings, Int. J. Bifurcation Chaos 9(9), 1815-1843, (1999).

20. Robinson, Clark, Dynamical systems. Stability, symbolic dynamics, and chaos, Studies in Advanced Mathematics. CRC Press, Boca Raton, FL, (1995).

21. David Sherwell and Vivien Visaya, Complexity of Multivalued Maps, World Academy of Science, Engineering and Technology 53, 717-720, (2011).

22. Sparrow, Colin, The Lorenz equations: bifurcations, chaos, and strange attractors, Applied Mathematical Sciences, 41. Springer-Verlag, New York-Berlin, (1982).

23. W. Tucker, The Lorenz attractor exists, Comptes Rendus de l'Académie des Scienes Série I. Mathématique 328, 1197-1202, (1999).

24. Visaya, Maria Vivien V., A lower estimate of the topological entropy from a one-dimensional reconstruction of time series, J. Math. Kyoto Univ. 46, no. 3, 637-655, (2006).

25. Yershov, M. P., Extensions of measures. Stochastic equations, Proceedings of the Second Japan-USSR Symposium on Probability Theory (Kyoto, 1972), pp. 516526. Lecture Notes in Math., Vol. 330, Springer, Berlin, (1973).

26. Ershov, M.P., Extensions of measures and stochastic equations, Theory of Probability and its Applications 19, no. 3, 431-444, (1974). 


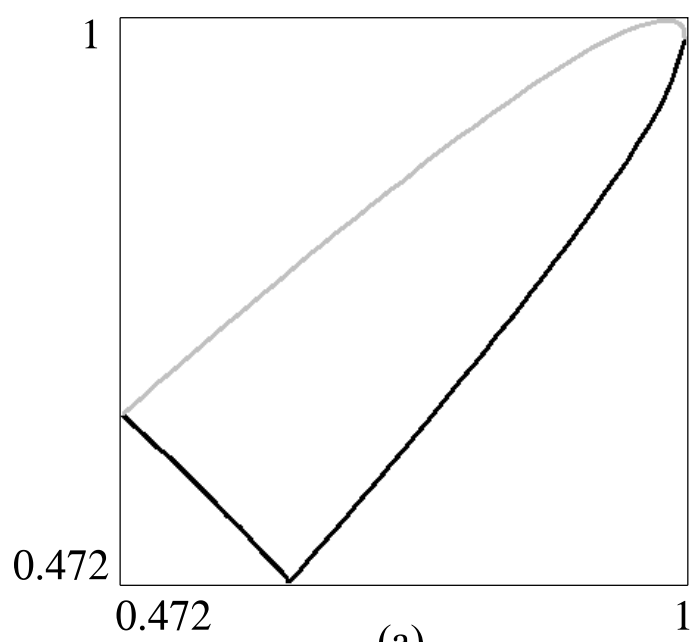

(a)

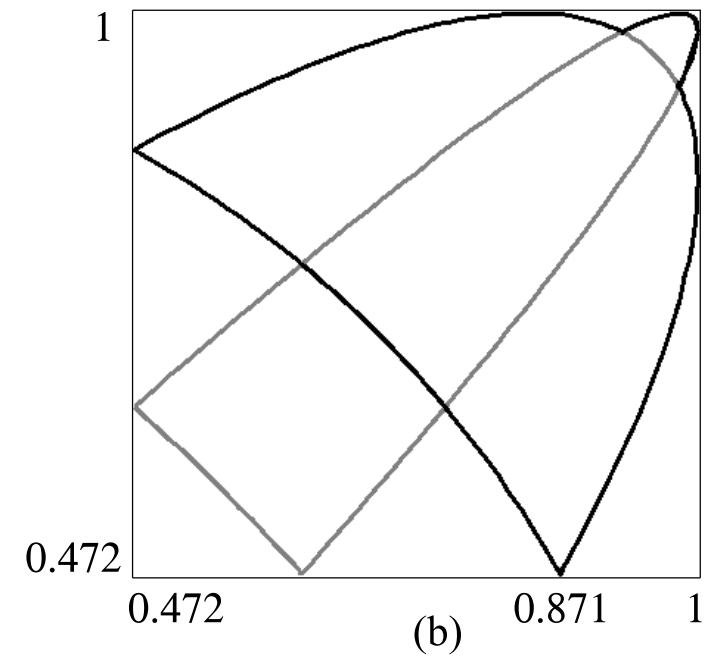

Fig. 9 Graph of consecutive maxima relation $T, \alpha=2.15722723$, and its third iterate $T^{3}$. 